PROCEEDINGS OF THE

AMERICAN MATHEMATICAL SOCIETY

Volume 130, Number 5, Pages 1257-1264

S 0002-9939(01)06418-8

Article electronically published on December 27, 2001

\title{
COUNTING GENERIC GENUS-0 CURVES ON HIRZEBRUCH SURFACES
}

\author{
HOLGER SPIELBERG
}

(Communicated by Mohan Ramachandran)

\begin{abstract}
Hirzebruch surfaces $F_{k}$ provide an excellent example to underline the fact that in general symplectic manifolds, Gromov-Witten invariants might well count curves in the boundary components of the moduli spaces. We use this example to explain in detail that the counting argument given by Batyrev for toric manifolds does not work.
\end{abstract}

\section{INTRODUCTION}

When Gromov-Witten invariants were first defined by Ruan and Tian RT95. for (weakly) monotone symplectic manifolds $(M, \omega)$, they counted certain smooth pseudo-holomorphic (rational) curves in $M$.

However, later it became clear that to extend the definition to general symplectic manifolds one had to take into account some contributions from nodal curves to obtain a symplectic invariant — this is now known as the virtual fundamental class construction (see [LT98], [FO99], Sie96]).

Although it is easy to see that one somehow has to deal with these singular curves to apply the general theory, it does not seem to be very clear what the singular curves actually contribute to the different Gromov-Witten invariants.

Moreover, Gromov-Witten invariants also enter as structure constants into the definition of the quantum cohomology ring. In Bat93, Batyrev gave an ad hoc definition of this ring for toric manifolds: the structure constants of Batyrev's ring count the same curves as Gromov-Witten invariants, but do not take into account the contributions of nodal curves.

In [Spi99], we have shown that for the threefold $\mathbb{P}_{\mathbb{C P}^{2}}(\mathcal{O}(3) \oplus \mathcal{O})$ Batyrev's ring has to be different from the (usual) quantum cohomology ring. However, this example is not very explicit and involves some complicated computations of Gromov-Witten invariants. A much easier example to explore in this context is that of Hirzebruch surfaces which also belong to the class of toric manifolds. Cox and Katz have pointed this out in [CK99, Example 11.2.5.2] in the case of $F_{2}=\mathbb{P}_{\mathbb{C P}^{1}}(\mathcal{O}(2) \oplus \mathcal{O})$ - here we will explain in detail how to obtain the Gromov-Witten invariants and the quantum cohomology ring of all Hirzebruch surfaces $F_{k}=\mathbb{P}_{\mathbb{C P}^{1}}(\mathcal{O}(k) \oplus \mathcal{O})$ and

Received by the editors October 6, 2000

1991 Mathematics Subject Classification. Primary 14N35; Secondary 53D45, 14H10, 14 M25.

Key words and phrases. Hirzebruch surfaces, quantum cohomology, Gromov-Witten invariants, toric manifolds. 
compare them to Batyrev's intersection product and quantum ring, respectively. In particular, we will point out precisely the contributions from nodal curves.

The main idea that makes the example so easy to study is that all pair Hirzebruch surfaces $F_{2 k}$ are in the same symplectic deformation class, as are all odd surfaces $F_{2 k+1}$. Hence their Gromov-Witten invariants and quantum cohomology rings all equal those of $F_{0} \cong \mathbb{C P}^{1} \times \mathbb{C P}^{1}$ (respectively $F_{1} \cong \widetilde{\mathbb{C P}^{2}}, \mathbb{C P}^{2}$ blown up at one point) up to isomorphism.

However, as complex manifolds, all Hirzebruch surfaces are equipped with an integrable complex structure, and those are all different. Therefore the holomorphic curves and their moduli spaces vary as well.

The article is structured as follows: We will first briefly review Hirzebruch surfaces and their constructions as toric manifolds. Here we will use Batyrev's notation, and we will also state the definition of his quantum ring in this context. We will then compute the Gromov-Witten invariants and the quantum cohomology ring of the Hirzebruch surfaces, and compare them to the Batyrev construction. Since the even and the odd cases are very similar we will restrict our attention to the former.

Notation conventions. For toric manifolds we will follow Batyrev's notation in Bat93 unless stated otherwise. However, we will denote Batyrev's quantum ring by Bat* and the usual quantum cohomology ring $Q H^{*}$. Multiplication in Bat* will be denoted by "о", while we use " $\star$ " for the multiplication in $Q H^{*}$; the multiplication in the usual (co)homology will be denoted by "." (or omitted).

\section{Description of Hirzebruch Surfaces as toric manifolds}

Hirzebruch surfaces $F_{k}$ are complex two-dimensional projective manifolds that are $\mathbb{C P}^{1}$-bundles over $\mathbb{C P}^{1}$ :

$$
F_{k}:=\mathbb{P}_{\mathbb{C P}^{1}}(\mathcal{O}(k) \oplus \mathcal{O}) .
$$

They also admit an effective action of a two-dimensional algebraic torus that is contained in $F_{k}$ as an open dense subset, i.e. they are toric manifolds. Their defining fan $\Sigma_{k}$ in $N=\mathbb{Z}^{2}$ with basis $e_{1}, e_{2}$ has the following set of one-dimensional cones:

$$
\begin{array}{ll}
v_{k, 1}=e_{1}, & v_{k, 3}=e_{2}, \\
v_{k, 2}=-e_{1}+k e_{2}, & v_{k, 4}=-e_{2} .
\end{array}
$$

The set of primitive collections is equal to $\mathfrak{P}\left(\Sigma_{k}\right)=\left\{\left\{v_{k, 1}, v_{k, 2}\right\},\left\{v_{k, 3}, v_{k, 4}\right\}\right\}$, and the set $R\left(\Sigma_{k}\right) \subset \mathbb{Z}^{4}$ of linear relations between the vectors $v_{k, i}$ is generated by the vectors

$$
\begin{aligned}
\lambda_{k, 1} & =(1,1,-k, 0), \\
\lambda_{k, 2} & =(0,0,1,1),
\end{aligned}
$$

that correspond under the isomorphism $R\left(\Sigma_{k}\right) \cong H_{2}\left(F_{k}, \mathbb{Z}\right)$ to the generators of the effective cone, that is the cone of classes that can be represented by holomorphic curves in $F_{k}$. The cohomology $H^{*}\left(F_{k}, \mathbb{Z}\right)$ is generated by the invariant divisor@

\footnotetext{
${ }^{1}$ We will omit the $k$ in the subscript, if no confusion can arise.
} 
$Z_{k, 1}, Z_{k, 2}, Z_{k, 3}$ and $Z_{k, 4}$ subject to relations described by the combinatorics of the fan:

$$
\begin{aligned}
H^{*}\left(F_{k}, \mathbb{Z}\right) & =\mathbb{C}\left[Z_{1}, \ldots Z_{4}\right] /\left\langle Z_{1}-Z_{2}, k Z_{2}+Z_{3}-Z_{4}, Z_{1} Z_{2}, Z_{3} Z_{4}\right\rangle \\
& =\mathbb{C}\left[Z_{1}, Z_{4}\right] /\left\langle Z_{1}^{2}, Z_{4}^{2}-k Z_{1} Z_{4}\right\rangle .
\end{aligned}
$$

The basis $\left\{Z_{k, 1}, Z_{k, 4}\right\}$ of $H^{2}\left(F_{k}, \mathbb{Z}\right)$ is dual to $\left(\lambda_{k, 1}, \lambda_{k, 2}\right)$ of $H_{2}\left(F_{k}, \mathbb{Z}\right)$, hence the classes $Z_{k, 1}$ and $Z_{k, 4}$ generate the Kähler cone of $F_{k}$. The Hirzebruch surfaces $F_{2 k}$ are all diffeomorphic to $F_{0}=\mathbb{C P}^{1} \times \mathbb{C P}^{1}$ with induced isomorphism $\varphi_{2 k}$ on the level of cohomology and degree-2 homology given by:

$$
\begin{aligned}
\varphi_{2 k}^{*}: H^{2}\left(F_{0}, \mathbb{Z}\right) & \stackrel{\sim}{\longrightarrow} H^{2}\left(F_{2 k}, \mathbb{Z}\right) \\
Z_{0,1} & \longmapsto Z_{2 k, 1} \\
Z_{0,4} & \longmapsto Z_{2 k, 4}-k Z_{2 k, 1}, \\
\left(\varphi_{2 k}\right)_{*}: H_{2}\left(F_{2 k}, \mathbb{Z}\right) & \stackrel{\sim}{\longrightarrow} H_{2}\left(F_{0}, \mathbb{Z}\right) \\
\lambda_{2 k, 1} & \longmapsto \lambda_{0,1}+k \lambda_{0,2} \\
\lambda_{2 k, 2} & \longmapsto \lambda_{0,2} .
\end{aligned}
$$

There are similar diffeomorphisms between $F_{2 k+1}$ and $F_{1}=\widetilde{\mathbb{C P}^{2}}$. In the following, we will only deal with the case of even Hirzebruch surfaces $F_{2 k}$ - the odd case $F_{2 k+1}$, however, is very similar.

\section{BATYREV'S INTERSECTION PRODUCT IN THE SPACE OF RATIONAL MAPS TO $F_{2 k}$}

In Bat93, Section 9], Batyrev considers the moduli space $\mathcal{I}_{\lambda}$ of holomorphic mappings $f: \mathbb{C P}^{1} \longrightarrow P_{\Sigma}$ to a toric manifold $P_{\Sigma}$ defined by a fan $\Sigma$ such that $f_{*}\left[\mathbb{C P}^{1}\right]=\lambda \in R(\Sigma) \cong H_{2}\left(P_{\Sigma}, \mathbb{Z}\right)$. A Riemann-Roch type argument gives the following expected (or virtual) dimension 2 of this moduli space:

$$
\operatorname{dim}_{\mathrm{vir}} \mathcal{I}_{\lambda}=2 \cdot\left(\operatorname{dim}_{\mathbb{C}} P_{\Sigma}+\left\langle c_{1}\left(P_{\Sigma}\right), \lambda\right\rangle\right)
$$

We should also remark here that the space $\mathcal{I}_{\lambda}$ has the same expected dimension as the corresponding moduli space of stable maps $\mathcal{M}_{0,3}^{\lambda}\left(P_{\Sigma}\right)$. Also note that $\mathcal{I}_{\lambda}$ can be considered the subspace of smooth curves in $\mathcal{M}_{0,3}^{\lambda}\left(P_{\Sigma}\right)$ by fixing three marked points $z_{1}, z_{2}, z_{3}$ on $\mathbb{C P}^{1}$.

There is a universal evaluation map ev $v_{\lambda}$ defined on $\mathcal{I}_{\lambda} \times \mathbb{C P}^{1}$ given by

$$
\begin{aligned}
\mathrm{ev}_{\lambda}: \mathcal{I}_{\lambda} \times \mathbb{C P}^{1} & \longrightarrow P_{\Sigma}, \\
(f, z) & \longmapsto f(z) .
\end{aligned}
$$

Let $z_{1}, \ldots, z_{m+1} \in \mathbb{C P}^{1}$ be $(m+1)$ pairwise different points, and define $\mathrm{ev}_{\lambda, i}:=$ $\left.\mathrm{ev}\right|_{\mathcal{I}_{\lambda} \times\left\{z_{i}\right\}}$ to be the restriction of ev to such a point in the second factor.

Let $\alpha_{1}, \ldots, \alpha_{m} \in H^{*}\left(P_{\Sigma}, \mathbb{Z}\right)$ be some cohomology classes of the toric manifold $P_{\Sigma}$, and $A_{1}, \ldots, A_{m} \subset P_{\Sigma}$ some cycles Poincaré dual to the classes $\alpha_{j}$ : $\left[A_{j}\right]=P . D .\left(\alpha_{j}\right)$. Then Batyrev's quantum intersection product in Batyrev's ring $\operatorname{Bat}^{*}\left(P_{\Sigma}, \mathbb{Z}\right)$ is defined by the requirement that

$$
\left\langle\alpha_{1} \circ \cdots \circ \alpha_{m}, B\right\rangle=\sum_{\lambda \in R(\Sigma)} e v_{\lambda, 1}^{-1}\left(A_{1}\right) \cdots e v_{\lambda, m}^{-1}\left(A_{m}\right) \cdot e v_{\lambda, m+1}^{-1}(B) q^{\lambda},
$$

\footnotetext{
${ }^{2}$ Note that in general, the actual dimension of the moduli space is bigger than the expected dimension; or that the moduli space might be empty although it has positive expected dimension.
} 
for all $B \in H_{*}\left(P_{\Sigma}, \mathbb{Z}\right)$, and linearity. Here the sum is over all $\lambda \in R(\Sigma)$ such that the intersection product in the sum is supposed to be of virtual dimension zero, i.e. such that

$$
\sum_{i=1}^{m} \operatorname{deg} \alpha_{i}-\operatorname{deg} B=2 \cdot \sum_{i=1}^{n} \lambda_{i}
$$

where $n$ is the number of one-dimensional cones in $\Sigma$.

Theorem 2.1 ([Bat93, Theorem 9.3]). Batyrev's ring Bat $^{*}\left(P_{\Sigma}, \mathbb{Z}\right)$ is generated by $Z_{1}, \ldots, Z_{n}$ subject to two types of relations:

(1) The same linear relations as in $Q H^{*}\left(P_{\Sigma}, \mathbb{Z}\right)$.

(2) For all classes $\lambda=\left(\lambda^{1}, \ldots, \lambda^{n}\right) \in R(\Sigma)$ with all $\lambda^{i} \geq 0$ non-negative, $Z_{1}^{\circ \lambda^{1}}$ 。 $\cdots \circ Z_{n}^{\circ \lambda^{n}}-q^{\lambda}$ is a relation.

Let us now restrict to the case of Hirzebruch surfaces, i.e. $\Sigma=\Sigma_{k}$ and $P_{\Sigma}=F_{k}$ :

Corollary 2.2. In the even case, Batyrev's ring for the Hirzebruch surfaces is given by the following presentation:

$$
\operatorname{Bat}^{*}\left(F_{2 k}, \mathbb{Z}\right)=\mathbb{Z}\left[Z_{2 k, 1}, Z_{2 k, 4}, q_{2 k, 1}, q_{2 k, 2}\right] /\left\langle\begin{array}{l}
Z_{2 k, 1}^{\circ 2} \circ Z_{2 k, 4}^{\circ 2 k}-q_{2 k, 1} q_{2 k, 2}^{2 k} \\
Z_{2 k, 4} \circ\left(Z_{2 k, 4}-2 k Z_{2 k, 1}\right)-q_{2}
\end{array}\right\rangle .
$$

\section{The quantum COHOMOlogy Ring of Hirzebruch Surfaces, AND THEIR COMPARISON TO BATYREV'S RING}

As mentioned earlier, we will restrict to the even Hirzebruch surfaces $F_{2 k}$. Remember that they are all in the same symplectic deformation class as $F_{0}=\mathbb{C P}^{1} \times$ $\mathbb{C P}^{1}$. The Gromov-Witten invariants of $\mathbb{C P}^{1}$ are well known (see for example RT95]):

Fact 3.1. The invariants

$$
\Phi_{0, m}^{r H, \mathbb{C P}^{1}}(\pi^{*} \beta ; \underbrace{H, \ldots, H}_{s \text {-times }}, \underbrace{1, \ldots, 1}_{(m-s) \text {-times }})
$$

with $\beta=P . D .[\mathrm{pt}] \in H^{*}\left(\overline{\mathcal{M}}_{0, m}\right)$ are equal to 1 if and only if $s=2 r+1$, and zero otherwise. Here $\pi: \mathcal{M}_{0, m}^{r H}\left(\mathbb{C P}^{1}\right) \longrightarrow \overline{\mathcal{M}}_{0, m}$ is the natural projection map, forgetting the map to $\mathbb{C P}^{1}$ and stabilizing.

Since the Gromov-Witten invariants of a product manifold are the product of Gromov-Witten invariants of the two factors, that is,

$$
\begin{aligned}
\Phi_{0, m}^{A+B, X \times Y}\left(\pi^{*}[p t] ;\right. & \left.\alpha_{1} \otimes \gamma_{1}, \ldots, \alpha_{m} \otimes \gamma_{m}\right) \\
& =\Phi_{0, m}^{A, X}\left(\pi^{*}[p t] ; \alpha_{1}, \ldots, \alpha_{m}\right) \cdot \Phi_{0, m}^{B, Y}\left(\pi^{*}[p t] ; \gamma_{1}, \ldots, \gamma_{m}\right),
\end{aligned}
$$

we hence know all Gromov-Witten invariants of $F_{0}=\mathbb{C P}^{1} \times \mathbb{C P}^{1}$. Remember that the quantum cohomology ring of $F_{k}$ equals $Q H^{*}\left(F_{k}, \mathbb{Q}\right):=H^{*}\left(F_{k}, \mathbb{Z}\right) \otimes$ $\mathbb{Q}\left[H_{2}\left(F_{k}, \mathbb{Z}\right)\right]$ with multiplication of two cohomology classe $\alpha, \beta \in H^{*}\left(F_{k}, \mathbb{Z}\right)$ given by

$$
\forall \gamma \in H^{*}\left(F_{k}, \mathbb{Z}\right):(\alpha \star \beta) \cup \gamma=\sum_{A \in H_{2}\left(F_{k}, \mathbb{Z}\right)} \Phi_{0,3}^{A, X}(\alpha, \beta, \gamma) q^{A}
$$

In general, multiplication between two elements in the quantum cohomology ring is then given by $\mathbb{Q}\left[H_{2}\left(F_{k}, \mathbb{Z}\right)\right]$-linear extension. 
So by Fact 3.1 and equation (3.1), the quantum cohomology ring of $F_{0}$ is equal to:

$$
Q H^{*}\left(F_{0}, \mathbb{Z}\right)=\mathbb{Z}\left[Z_{0,1}, Z_{0,4}, q_{0,1}, q_{0,2}\right] /\left\langle Z_{0,1}^{2}-q_{0,1}, Z_{0,4}^{2}-q_{0,2}\right\rangle
$$

where we have written $q_{0, i}=q^{\lambda_{0, i}}$ for short 3

Remark 3.2. Note that for $F_{0}=\mathbb{C P}^{1} \times \mathbb{C P}^{1}$ (as well as for $F_{1}$ ), the Gromov-Witten invariants are equal to Batyrev's intersection products (cf. [Bat93, Definition 9.2]). This is due to the fact that $F_{0}$ and $F_{1}$ are Fano - in this case, the space of nodal curves is too small to contribute to the Gromov-Witten invariants.

In the following we will omit the class $\beta \in H^{*}\left(\overline{\mathcal{M}}_{0, m}\right)$ in the Gromov-Witten invariants, always assuming that $\beta=P . D .[\mathrm{pt}]$.

Corollary 3.3. The quantum cohomology ring of the Hirzebruch surface $F_{2 k}$ is given by

$$
Q H^{*}\left(F_{2 k}, \mathbb{Z}\right)=\mathbb{Z}\left[Z_{2 k, 1}, Z_{2 k, 4}, q_{2 k, 1}, q_{2 k, 2}\right] /\left\langle\begin{array}{l}
Z_{2 k, 1}^{\star 2}-q_{2 k, 1} q_{2 k, 2}^{-k} \\
\left(Z_{2 k, 4}-k Z_{2 k, 1}\right)^{\star 2}-q_{2 k, 2}
\end{array}\right\rangle .
$$

Proof. We just have to apply the isomorphisms (1.1) and (1.2):

$$
Z_{2 k, 1}^{\star 2}-q_{2 k, 1} q_{2 k, 2}^{-k}=\varphi_{2 k}^{*}\left(Z_{0,1}^{\star 2}-q_{0,1} q_{0,2}^{k} q_{0,2}^{-k}\right)=0
$$

and similarly

$$
\left(Z_{2 k, 4}-k Z_{2 k, 1}\right)^{\star 2}-q_{2 k, 2}=\varphi_{2 k}^{*}\left(\left(Z_{0,4}+k Z_{0,1}-k Z_{0,1}\right)^{\star 2}-q_{0,2}\right)=0 .
$$

It is now easy to see that the above presentation for the quantum cohomology ring and the presentation for Batyrev's ring given in Corollary 2.2 define two different rings.

In the remaining part of the article we will now compute the relations in Batyrev's ring, but using quantum multiplication to illustrate for which homology classes nodal curves contribute to the Gromov-Witten invariants. The products we want to compute are

$$
Z_{2 k, 3} \star Z_{2 k, 4} \quad \text { and } \quad Z_{2 k, 1} \star Z_{2 k, 2} \star Z_{2 k, 4}^{\star 2 k} \text {. }
$$

Hence we have to determine the following invariants:

$$
\Phi_{0,3+2 k}^{\lambda, F_{2 k}}(Z_{2 k, 1}, Z_{2 k, 2}, \underbrace{Z_{2 k, 4}, \ldots, Z_{2 k, 4}}_{2 k \text {-times }}, \gamma), \quad \Phi_{0,3}^{\lambda, F_{2 k}}\left(Z_{2 k, 3}, Z_{2 k, 4}, \gamma\right) .
$$

Note that for any class $\lambda \in R\left(\Sigma_{2 k}\right),\left\langle c_{1}\left(F_{2 k}\right), \lambda\right\rangle \equiv 0 \bmod 2$ is even. Thus we only have to consider $\gamma=1$ or $\gamma=Z_{2 k, 1} Z_{2 k, 4}=P . D .([\mathrm{pt}])$.

Lemma 3.4. The Gromov-Witten invariants $\Phi_{0,3}^{\lambda, F_{2 k}}\left(Z_{2 k, 3}, Z_{2 k, 4}, \gamma\right)$ are given by:

$$
\begin{aligned}
\Phi_{0,3}^{\lambda, F_{2 k}}\left(Z_{3}, Z_{4}, 1\right) & =0 \quad \text { for all } \lambda \in R(\Sigma), \\
\Phi_{0,3}^{\lambda, F_{2 k}}\left(Z_{3}, Z_{4}, Z_{1} Z_{4}\right) & = \begin{cases}1 & \text { if } \lambda=\lambda_{2 k, 2}, \\
-k^{2} & \text { if } \lambda=\lambda_{2 k, 1}+k \lambda_{2 k, 2}, \\
0 & \text { otherwise. }\end{cases}
\end{aligned}
$$

\footnotetext{
${ }^{3}$ Note that it is important to keep track of the mapping $H_{2}(X, \mathbb{Z}) \longrightarrow Q H^{*}(X, \mathbb{Z})$. Otherwise the statements become void since as abstract rings, all rings under consideration, whether quantum cohomology or Batyrev's, coincide: they are all free rings generated by $H^{2}\left(F_{k}, \mathbb{Z}\right)$.
} 
Proof. For the first line, remember that $\Phi_{0,3}^{\lambda, X}(A, B, 1)=A \cdot B$ if $\lambda=0$, and zero otherwise. But here we also have that $Z_{2 k, 3} \cdot Z_{2 k, 4}=0$. For the second line, using the properties of the isomorphisms $\varphi_{2 k}^{*}$ and $\left(\varphi_{2 k}\right)_{*}$ we obtain that

$$
\begin{aligned}
\Phi_{0,3}^{r \lambda_{2 k, 1}+s \lambda_{2 k, 2}, F_{2 k}}\left(Z_{2 k, 3}, Z_{2 k, 4}, Z_{2 k, 1} Z_{2 k, 4}\right) \\
=\Phi_{0,3}^{r \lambda_{0,1}+(s-k r) \lambda_{0,2}, F_{0}}\left(Z_{0,4}-k Z_{0,1}, Z_{0,4}+k Z_{0,1}, Z_{0,1} Z_{0,4}\right) \\
=\Phi_{0,3}^{r H, \mathbb{C P}^{1}}(1,1, H) \cdot \Phi_{0,3}^{(s-k r) H, \mathbb{C P}^{1}}(H, H, H) \\
\quad-k^{2} \Phi_{0,3}^{r H, \mathbb{C P}^{1}}(H, H, H) \cdot \Phi_{0,3}^{(s-k r) H, \mathbb{C P}^{1}}(1,1, H) \\
=\delta_{0, r} \cdot \delta_{1, s}-k^{2} \delta_{1, r} \cdot \delta_{s, k} .
\end{aligned}
$$

For the last line we have used the properties of the Gromov-Witten invariants of $\mathbb{C P}^{1}$ (Fact 3.1).

Corollary 3.5. For the Hirzebruch surface $F_{2 k}$, the quantum product $Z_{2 k, 3} \star Z_{2 k, 4}$ equals

$$
Z_{2 k, 3} \star Z_{2 k, 4}=q_{2 k, 2}-k^{2} q_{2 k, 1} q_{2 k, 2}^{k},
$$

while Batyrev's product yields

$$
Z_{2 k, 3} \circ Z_{2 k, 4}=q_{2 k, 2} .
$$

Remark 3.6. It is easy to see that holomorphic curves in the class $\lambda:=\lambda_{2 k, 2}+k \lambda_{2 k, 1}$ cannot be smooth. In fact, $\lambda=(1,1,-k, k)$, hence any smooth curve of that class would have to lie in the divisor $Z_{2 k, 3}$. However $Z_{2 k, 3}$ is Poincaré dual to $\lambda_{2 k, 1}$, so any class lying in the divisor $Z_{2 k, 3}$ has homology class a multiple of $\lambda_{2 k, 1}$, which is a contradiction. Hence the contribution $-k^{2} q_{2 k, 1} q_{2 k, 2}^{k}$ comes from nodal curves.

Lemma 3.7. The invariants of the form $\Phi_{0,3+2 k}^{\lambda, F_{2 k}}(Z_{2 k, 1}, Z_{2 k, 2}, \underbrace{Z_{2 k, 4}, \ldots, Z_{2 k, 4}}_{2 k-\text { times }}, 1)$ are zero except for the following:

$\Phi_{0,3+2 k}^{r \lambda_{2 k, 1}+((k-1)(r+1)+1) \lambda_{2 k, 2}, F_{2 k}}(Z_{2 k, 1}, Z_{2 k, 2}, \underbrace{Z_{2 k, 4}, \ldots, Z_{2 k, 4}}_{2 k-\text { times }}, 1)=\left(\begin{array}{c}2 k \\ 2 r-1\end{array}\right) k^{2 r-1}$ where $r=1, \ldots, k$.

Proof. Let us write $\lambda=r \lambda_{2 k, 1}+s \lambda_{2 k, 2}$. By applying Fact 3.1 and equation (3.1) we obtain

$$
\begin{aligned}
\Phi_{0,3+2 k}^{r \lambda_{2 k, 1}+s \lambda_{2 k, 2}, F_{2 k}}(Z_{2 k, 1}, Z_{2 k, 2}, \underbrace{Z_{2 k, 4}, \ldots, Z_{2 k, 4}}_{2 k}, 1) \\
=\Phi_{0,3+2 k}^{r \lambda_{0,1}+(s-k r) \lambda_{0,2}, F_{0}}(Z_{0,1}, Z_{0,1}, \underbrace{Z_{0,4}+k Z_{0,1}, \ldots, Z_{0,4}+k Z_{0,1}}_{2 k}, 1) \\
=\sum_{i=0}^{2 k}\left(\begin{array}{c}
2 k \\
i
\end{array}\right) k^{i} \Phi_{0,3+2 k}^{r H, \mathbb{C P}^{1}}(\underbrace{H, \ldots, H}_{i+2}, \underbrace{1, \ldots, 1}_{2 k+1-i}) \Phi_{0,3+2 k}^{(s-k r) H, \mathbb{C P}^{1}}(\underbrace{1, \ldots, 1}_{3+i}, \underbrace{H, \ldots, H}_{2 k-i}) \\
=\left\{\begin{array}{cc}
\left(\begin{array}{c}
2 k \\
2 r-1
\end{array}\right) k^{2 r-1} \Phi_{0,3+2 k}^{(s-k r) H, \mathbb{C P}^{1}}(\underbrace{1, \ldots, 1}_{2 r+2}, \underbrace{H, \ldots, H}_{2 k-2 r+1}), & 0 \leq 2 r-1 \leq 2 k, \\
0, & \text { otherwise, }
\end{array}\right.
\end{aligned}
$$




$$
= \begin{cases}\left(\begin{array}{c}
2 k \\
2 r-1
\end{array}\right) k^{2 r-1}, & 0 \leq 2 r-1 \leq 2 k, s=(k-1)(r+1)+1, \\
0, & \text { otherwise }\end{cases}
$$

which proves the lemma.

Lemma 3.8. The invariants $\Phi_{0,3+2 k}^{\lambda, F_{2 k}}(Z_{2 k, 1}, Z_{2 k, 2}, \underbrace{Z_{2 k, 4}, \ldots, Z_{2 k, 4}}_{2 k-\text { times }}, P . D .[\mathrm{pt}])$ are all zero except for the following:

$$
\Phi_{0,3+2 k}^{r \lambda_{2 k, 1}+((k-1)(r+1)+2) \lambda_{2 k, 2}, F_{2 k}}(Z_{2 k, 1}, Z_{2 k, 2}, \underbrace{Z_{2 k, 4}, \ldots, Z_{2 k, 4}}_{2 k-\text { times }}, Z_{2 k, 1} Z_{2 k, 4})
$$

which equal $\left(\begin{array}{c}2 k \\ 2 r-2\end{array}\right) k^{2 r-2}$. Here $r=1, \ldots, k+1$.

Proof. Similar to the proof of Lemma 3.7.

Corollary 3.9. For the Hirzebruch surface $F_{2 k}$, the quantum product $Z_{2 k, 1} \star Z_{2 k, 2} \star$ $Z_{2 k, 4}^{\star 2 k}$ equals

$$
\begin{aligned}
Z_{2 k, 1} \star Z_{2 k, 2} \star Z_{2 k, 4}^{\star 2 k}= & \sum_{r=1}^{k}\left(\begin{array}{c}
2 k \\
2 r-1
\end{array}\right) k^{2 r-1} q_{2 k, 1}^{r} q_{2 k, 2}^{(k-1)(r+1)+1} Z_{2 k, 1} Z_{2 k, 4} \\
& +\sum_{r=1}^{k+1}\left(\begin{array}{c}
2 k \\
2 r-2
\end{array}\right) k^{2 r-2} q_{2 k, 1}^{r} q_{2 k, 2}^{(k-1)(r+1)+2},
\end{aligned}
$$

while Batyrev's product yields

$$
Z_{2 k, 1} \circ Z_{2 k, 2} \circ Z_{2 k, 4}^{\circ 2 k}=q_{2 k, 1} q_{2 k, 2}^{2 k} .
$$

Remark 3.10. Note that as for the product $Z_{2 k, 3} \star Z_{2 k, 4}$ in Corollary 3.5, Batyrev's intersection product is included in the terms entering the quantum product based on Gromov-Witten invariants. This is of course remarkable since it shows - at least for the Hirzebruch surfaces and for non-negative classes $\lambda$ - that the boundary components of $\mathcal{M}_{0, m}^{\lambda}\left(F_{2 k}\right)$ do not influence the corresponding Gromov-Witten invariant

$$
\Phi_{0, \lambda^{1}+\cdots+\lambda^{n}+1}^{\lambda, F_{2 k}}(\underbrace{Z_{2 k, 1}, \ldots, Z_{2 k, 1}}_{\lambda^{1}}, \ldots, \underbrace{Z_{2 k, n}, \ldots, Z_{2 k, n}}_{\lambda^{n}}, \gamma) .
$$

However, the boundary components of the moduli spaces enter nonetheless through the invariants

$$
\Phi_{0, \lambda^{1}+\cdots+\lambda^{n}+1}^{\lambda^{\prime}, F_{2 k}}(\underbrace{Z_{2 k, 1}, \ldots, Z_{2 k, 1}}_{\lambda^{1}}, \ldots, \underbrace{Z_{2 k, n}, \ldots, Z_{2 k, n}}_{\lambda^{n}}, \gamma) \neq 0
$$

where $\lambda \neq \lambda^{\prime}$.

\section{ACKNOWLEDGEMENTS}

I thank Michèle Audin and Bernd Siebert for fruitful discussions. I also thank the Max Planck Institute for Mathematics in the Sciences, Leipzig, for its hospitality. 


\section{REFERENCES}

[Bat93] Victor V. Batyrev. Quantum cohomology rings of toric manifolds. Astérisque, 218:9-34, 1993. MR 95b:32034

[CM00] Laura Costa and Rosa M. Miró-Roig. The Leray quantum relation for a class of non-Fano toric varieties. Preprint.

[CK99] David A. Cox and Sheldon Katz. Mirror Symmetry and Algebraic Geometry. Mathematical Surveys and Monographs 68, Amer. Math. Soc., 1999. MR 2000d:14048

[FO99] K. Fukaya and K. Ono. Arnold conjecture and Gromov-Witten invariants. Topology, 38(5):933-1048, 1999. MR 2000j:53116

[Giv98] Alexander Givental. A mirror theorem for toric complete intersections. Topological field theory, primitive forms and related topics, Kyoto, 1996, pages 141-175, Prog. Math., 160, Birkhäuser, 1998. MR 2000a:14063

[Gro85] M. Gromov. Pseudo-holomorphic curves in symplectic geometry. Invent. Math., 82:307347, 1985. MR 87j:53053

[LT98] Jun Li and Gang Tian. Virtual moduli cycles and Gromov-Witten invariants of general symplectic manifolds. Topics in symplectic 4-manifolds, Irvine, 1996, pages 47-83, International Press, 1998. MR 2000d:53137

[RT95] Yongbin Ruan and Gang Tian. A mathematical theory of quantum cohomology. J. Diff. Geom., 42(2):259-367, 1995. MR 96m:58033

[Sie96] Bernd Siebert. Gromov-Witten invariants for general symplectic manifolds. Preprint dgga/9608005, LANL preprint server, August 1996.

[Sie99] Bernd Siebert. An update on (small) quantum cohomology. Mirror symmetry III (Montreal, PQ, 1995), Number 10 in AMS/IP Stud. Adv. Math., pages 279-312, Amer. Math. Soc., 1999. MR 2000d:14060

[Spi99] Holger Spielberg. The Gromov-Witten invariants of symplectic toric manifolds, and their quantum cohomology ring. C. R. Acad. Sci. Paris, Série I, 329(8):699-704, 1999. MR 2000j:14087

Departamento de Matemática, Instituto Superior Técnico, Av. Rovisco Pais, 1049001 Lisboa, Portugal

E-mail address: Spielberg@member.ams.org 